\title{
Metastable States in Two-Lane Traffic Flow Models With Slow-To-Start Rule
}

\author{
Najem Moussa ${ }^{1,2}$ * \\ ${ }^{1}$ Faculté Polydisciplinaire, El Jadida, Morocco \\ ${ }^{2}$ LPMC, Faculté des Sciences, El Jadida, Morocco
}

July 26, 2018

\begin{abstract}
Using computer simulations, we show that metastable states still occur in two-lane traffic models with slow to start rules. However, these metastable states no longer exist in systems where aggressive drivers (which do not look back before changing lanes) are present. Indeed, the presence of only one aggressive driver in the circuit, triggers the breakdown of the high flow states. In these systems, the steady state is unique and its relaxation dynamics should depend on the lane changing probability $p_{c h}$ and the number of aggressive drivers present in the circuit. It is found also that the relaxation time $\tau$ diverges as the form of a power-law : $\tau \propto p_{c h}^{-\beta}, \beta=1$.
\end{abstract}

PACS. 02.50.-Ey Stochastic processes 05.45.-a Nonlinear dynamics and nonlinear dynamic systems $45.70 . V n$ Granular models of complex systems; traffic flow 89.40.+k Transportation

*e-mail: najemmoussa@yahoo.fr 
Recently, cellular automata (CA) traffic models are used enormously in order to understand the complex dynamic behavior of the traffic in roadways (see the review [1]). In CA, time and space are discrete. The space is represented as a uniform lattice of cells with finite number of states, subject to a uniform set of rules, which drives the behavior of the system. These rules compute the state of a particular cell as a function of its previous state and the state of the neighboring cells. The most popular CA model for traffic flow on one-lane roadway is the NaSch model [2]. Despite its simplicity, the model is capable of capturing some essential features observed in realistic traffic like density waves or spontaneous formation of traffic jams. To describe more complex situations such as multi-lane traffic, extensions of the NaSch model have been proposed where additional rules are added for lane changing cars.

Barlovic et al [3] found metastable states in their velocity dependent randomization (VDR) which is an extension of the NaSch model. The one-lane VDR model belongs to the class of CA models with "slow-to-start" rules. These models show an hysteresis effect which is a consequence of the non-unique dependence of the flow on the density. The above characteristic behavior of traffic flow is also observed in two-lane traffic models. Indeed, Awazu [4] showed the appearance of several branches and hysteresis in the relation between traffic flow and car density.

To establish the existence of metastable states, Barlovic et al started their simulations of the VDR model from two different initial configurations, the megajam and the homogeneous state. The megajam consists of one large compact cluster of standing cars. In the homogeneous state, cars are distributed periodically with equal constant gap between successive cars (with one lager gap for incommensurate densities). If the initial configuration is homogeneous, one obtains the upper branch, for some interval of densities $\left[\rho_{1}, \rho_{2}\right]$, in which each car moves freely with maximal velocity (see figure 1 ). This upper branch is metastable with an extremely long life-time. If the initial configuration is megajam, one obtains the lower branch which is phase separated. The phase separated state consists of a large jam (jammed region) and a free-flow region where each car moves freely. It is known that the lifetime of the metastable states depends on the system length L. Yet, the simulation results indicate that $\Delta \rho=\rho_{1}-\rho_{2}$ decreases with larger system sizes and is expected to vanish for $L \rightarrow \infty$, i.e. the jammed branch is stable in that limit. Therefore the non-unique behaviour of the fundamental diagram is only observable if finite system sizes are considered (see [1] page 
93).

As vehicular traffic usually evolved in multi-lane roads, some interesting question is not yet studied. Does multi-lane version of the VDR model always exhibits metastable states?

The NaSch model with VDR rule is a one-dimensional probabilistic CA which consists of $N$ cars moving on a one-dimensional lattice of $L$ cells with periodic boundary conditions (the number of vehicles is conserved). Each cell is either empty, or occupied by just one vehicle with velocity $v=1,2, \ldots, v_{\max }$. We denote by $x_{k}$ and $v_{k}$ the position and the velocity of the $k$ th car at time $t$ respectively. The number of empty cells in front of the $k t h$ car is denoted by $d_{k}=x_{k+1}-x_{k}-1$ and called hereafter as the gap. Space and time are discrete. At each discrete time-step $t \rightarrow t+1$ the system update is performed in parallel for all cars according to the following four subrules :

$R_{1}$ : VDR, $p\left(v_{k}\right)=p_{0}$ for $v_{k}=0$ and $p\left(v_{k}\right)=p$ for $v_{k}>0$.

$R_{2}$ : Acceleration, $v_{k} \leftarrow \min \left(v_{k}+1, v_{\max }\right)$.

$R_{3}$ : Slowing down, $v_{k} \leftarrow \min \left(v_{k}, d_{k}\right)$.

$R_{4}$ : Randomization, $v_{k} \leftarrow \max \left(v_{k}-1,0\right)$ with probability $p\left(v_{k}\right)$.

$R_{5}$ : Motion, the car is moved forward according to its new velocity, $x_{k} \leftarrow$ $x_{k}+v_{k}$.

In two-lane traffic models, lane changing of vehicles are performed according to some additional rules [5-9]. In this paper, we shall adopt the symmetric exchange rules which are defined by the following criteria [6]:

1. $\min \left(v_{k}+1, v_{\max }\right)>d_{k}$

2. $d_{k, \text { other }}>d_{k}$ and $d_{k, \text { back }}>l_{\text {back }}$

3. $p_{\text {ch }}>\operatorname{rand}()$

Here $d_{k, \text { other }}$ (resp. $d_{k, \text { back }}$ ) denotes the gap on the target lane in front of (resp. behind) the car that wants to change lanes. Two different formulas are assigned to the parameter $l_{\text {back }}$. For aggressive drivers, i.e., vehicles which do not look back before changing lanes, we choose $l_{\text {back }}=0$. For careful drivers, i.e., vehicles which respect the safety criterion, we choose $l_{b a c k}=v_{o}^{b}+1$, where $v_{o}^{b}$ is the velocity of the following car in the target lane. Finally, $p_{c h}$ is the lane-changing probability and $\operatorname{rand}()$ stands for a random number between 0 and 1. Hereafter, we shall denote by $\operatorname{VDRM}_{N_{a}}$ the two-lane VDR traffic models where $N_{a}$ represents the number of aggressive drivers present in the circuit. 
The update in the two-lane model is divided into two sub-steps: in one substep, cars may change lanes in parallel following the above lane changing rules and in the other sub-step each car may move effectively by the forward movement rules as in the single-lane traffic.

We performed computer simulations of the two-lane model with the following parameters, $\left(p_{0}=0.01, p=0.7\right.$ and $\left.v_{\max }=5\right)$. The size of the lattice is given by $L=1000$. Starting from an initial configuration (homogeneous or megajam) the system evolved in time steps with respect to the above dynamical rules. For each simulation run, we discarded some number $\left(t_{d i s}\right)$ of time steps and we performed averages of the flow over $t_{a v}=50000$ time steps. The duration of each run is " $t_{d i s}+t_{a v}$ ". The procedure is then repeated for a number 100 of different realizations of the homogeneous (or megajam) initial configurations. The average over all the different realizations gives a mean value of the flow.

Figure 1 illustrated the variation of the flow $J$, in two-lane VDR traffic models, as a function of the density of cars and for different values of the discarded time $t_{d i s}$. We noticed that the flow in both lanes are equal since symmetric lane changing are considered. First, we shall consider the case where only one aggressive driver is present in the circuit. So, if the homogeneous initial state is used, a higher branch of the flow is observed for some interval of densities $\left[\rho_{1}, \rho_{2}\right]$ whenever $t_{d i s}$ is small enough. When increasing enough $t_{d i s}$, the high branch interval diminished and disappeared completely at certain limit of $t_{d i s}$. Notice that this phenomena occurred also for the NS model with very small randomization $p$. In contrast, the upper branch in the fundamental diagram of the $\mathrm{VDRM}_{0}$ does not change when one increases the time $t_{d i s}$ (Fig. 1). This shows clearly that the hysteresis exist in the fundamental diagram of the $\mathrm{VDRM}_{0}$ model but not in the one of the $\mathrm{VDRM}_{1}$.

To clarify more the above results, we shall consider the time evolution of the flow for some fixed density $\rho=0.12\left(\rho_{1}<\rho<\rho_{2}\right)$ and for the homogeneous and megajam initial states (Fig. 2). It is shown that in contrast to the $\mathrm{VDRM}_{0}$, where the homogeneous state is metastable with an extremely long life-time, this state does not exist in $\mathrm{VDRM}_{1}$. Yet, in this later, the flow corresponding to the homogeneous initial configuration decreases with time until reaching the value corresponding to the megajam initial configuration. The breakdown of the homogeneous structure in the two lanes is due to the occurrence of stopped cars provoked by the abrupt lane changing of the aggressive driver. Figure 3 shows the evolution of the density of stopped cars in the lanes when starting from the initial homogeneous state. In $\mathrm{VDRM}_{0}$, 
no stopped cars exist in the circuit because all cars respect the safety criteria of lane changing. However, in $\mathrm{VDRM}_{1}$, the density increases with time until it reaches a stationary value. Stopped cars act as perturbations for the free flow region and as such trigger the breakdown of the high flow states.

In figure 4 , we show the cluster size distribution in the steady state of $\mathrm{VDRM}_{1}$ for different lane-changing probability $p_{c h}$. The cluster means here a string of successive stopped cars in a single lane of the two-lane model, i.e. we are considering only compact jams in a single lane. As the symmetric lane-changing rules are considered here, the cluster sizes distribution in the two lanes must be equal. We observe from figure 4 the bimodal nature of the cluster size distribution as $p_{c h} \simeq 1$. Large clusters appear in the lanes but there are by far many more small-sized clusters than large ones. Furthermore, with decreasing $p_{c h}$, the probability of small clusters increases while that of large clusters diminishes. If $p_{c h}=0$, which corresponds to the single lane VDR model, almost all cars are congested in one large cluster with the exception of a few isolated cars. This is the well known phase separated state.

In this section, we shall investigate the relaxation dynamics of $\operatorname{VDRM}_{N_{a}}$ for different values of $N_{a}$ and $p_{c h}$, when starting from the homogeneous initial condition. This is done by plotting the time evolutions of the flow and computing their relaxation times. Hence, the greater is the number of aggressive drivers, the faster is the system relaxation (Fig. 5). When decreasing the probability of lane changing $p_{c h}$, one sees that the equilibration is delayed. Indeed, in this case, the abrupt lane changes of aggressive cars become less frequent and the number of stopped cars becomes small (Fig. 6).

To study numerically the relaxation time corresponding to an observable $A$ we shall use the nonlinear relaxation function [10]:

$$
\phi(t)=[A(t)-A(\infty)] /[A(0)-A(\infty)]
$$

The corresponding nonlinear relaxation time

$$
\tau=\int_{0}^{\infty} \phi(t) d t .
$$

The condition that the system is well equilibrated is

$$
t_{M_{0}} \gg \tau
$$

where $M_{0}$ is the number of Monte Carlo steps that have to be excluded in the averaging of the observable $A$. In figure 7 , we plotted the variation of 
the relaxation time $\tau$ of the observable $J$ near the limit $p_{c h} \rightarrow 0$. As a result, the relaxation time is found to diverge as $p_{c h} \rightarrow 0$. Moreover, we see that $\tau$ follows a power law behavior of the form,

$$
\tau \propto p_{c h}^{-\beta}
$$

Except for some minor fluctuations, the dynamic exponent $\beta$ remains unchanged when varying the number of aggressive drivers present in the circuit. For example, $\beta \approx 0,9798 \pm 0,0290$ for $N_{a}=1$ and $\beta \approx 0,9801 \pm 0,0315$ for $N_{a}=3$. Assuming that the parameter $p_{c h}$ is rate of transition for the dynamics of the model, and as it was demonstrated in Ref. [11], the exponent $\beta$ is expected to be theoretically equal to one.

In summary, we have shown that the presence of aggressive drivers in the circuit breakdowns the state of high traffic flow. In theses systems neither phase separation nor metastability can occur and a new stationary state takes place. Indeed, the abrupt lane changing of aggressive drivers force the succeeding cars on the destination lane to decelerate enough; leading therefore to the occurrence of stopped cars and then the formation of jams.

In the NS model, the cluster sizes distribution decreases exponentially while in $\mathrm{VDRM}_{0}$, it should depend on the initial state. Yet, for some density $\rho$ in the hysteresis region $\left(\rho_{1}<\rho<\rho_{2}\right)$, no clusters appear in the metastable homogeneous state. However, a big cluster persists in the phase separated state. Nevertheless, in $\operatorname{VDRM}_{N_{a}}$ where $N_{a} \neq 0$, the stationary state is composed by small and big clusters. The distribution of these clusters should depend on the lane-changing probability $p_{c h}$. As $p_{c h}$ decreases the relaxation time $\tau$ of the system increases and diverges at the limit $p_{c h} \rightarrow 0$. The relaxation behaviour follows a power law behavior of the form, $\tau \propto p_{c h}^{-\beta}, \beta=1$ ). 


\section{References}

[1] D. Chowdhury, L. Santen and A. Schadschneider, Phys. Rep. 329199 (2000).

[2] K. Nagel and M. Schreckenberg, J. Phys. (France) I, 2, 2221 (1992).

[3] R. Barlovic, L. Santen, A. Schadschneider and M. Schreckenberg, Eur. Phys. J. B, 5, 793 (1998).

[4] A. Awazu, J. Phys. Soc. Jpn 67, 1071 (1998).

[5] M. Rickert, K. Nagel, M. Schreckenberg and A. Latour, Physica A 231, 534 (1996).

[6] D. Chowdhury, D. E. Wolf and M. Schreckenberg, Physica A 235, 417 (1997).

[7] W. Knospe, L. Santen, A. Schadschneider and M. Schreckenberg, Physica A 265, 614 (1999).

[8] W. Knospe, L. Santen, A. Schadschneider and M. Schreckenberg, J. Phys. A: Math. Gen. 35, 3369 (2002).

[9] N. Moussa and A.K. Daoudia, Eur. Phys. J. B, 31, 413 (2003).

[10] K. Binder, Rep. Prog. Phys. 60, 487 (1997).

[11] N. Moussa, Phys. Rev. E 71, 026124 (2005). 


\section{Figures captions}

Figure 1. Illustration of the variation of the flow $J$ in the twolane VDR traffic models as a function of the density of cars and for different values of the discarded time $t_{d i s}\left(p_{c h}=0.10\right)$.

Figure 2. Time evolutions of the flow for homogeneous and megajam initial states $\left(\rho=0.12\right.$ and $\left.p_{c h}=0.10\right)$.

Figure 3. Time evolution of the density of stopped cars when starting from the initial homogeneous state $\left(\rho=0.12, N_{a}=1\right.$ and $\left.p_{c h}=0.10\right)$.

Figure 4. The cluster size distribution in the steady state for different lane-changing probability $p_{c h}\left(\rho=0.12\right.$ and $\left.N_{a}=1\right)$.

Figure 5. Time evolutions of the flow and the density of stopped cars, when starting from homogeneous initial configuration, for several values of $N_{a}\left(\rho=0.12\right.$ and $\left.p_{c h}=0.10\right)$.

Figure 6. Time evolutions of the flow and the density of stopped cars, when starting from homogeneous initial configuration, for several values of $p_{c h}\left(\rho=0.12\right.$ and $\left.N_{a}=1\right)$.

Figure 7. Variations of the relaxation time $\tau$ near the limit $p_{c h} \rightarrow 0(\rho=0.12)$. 


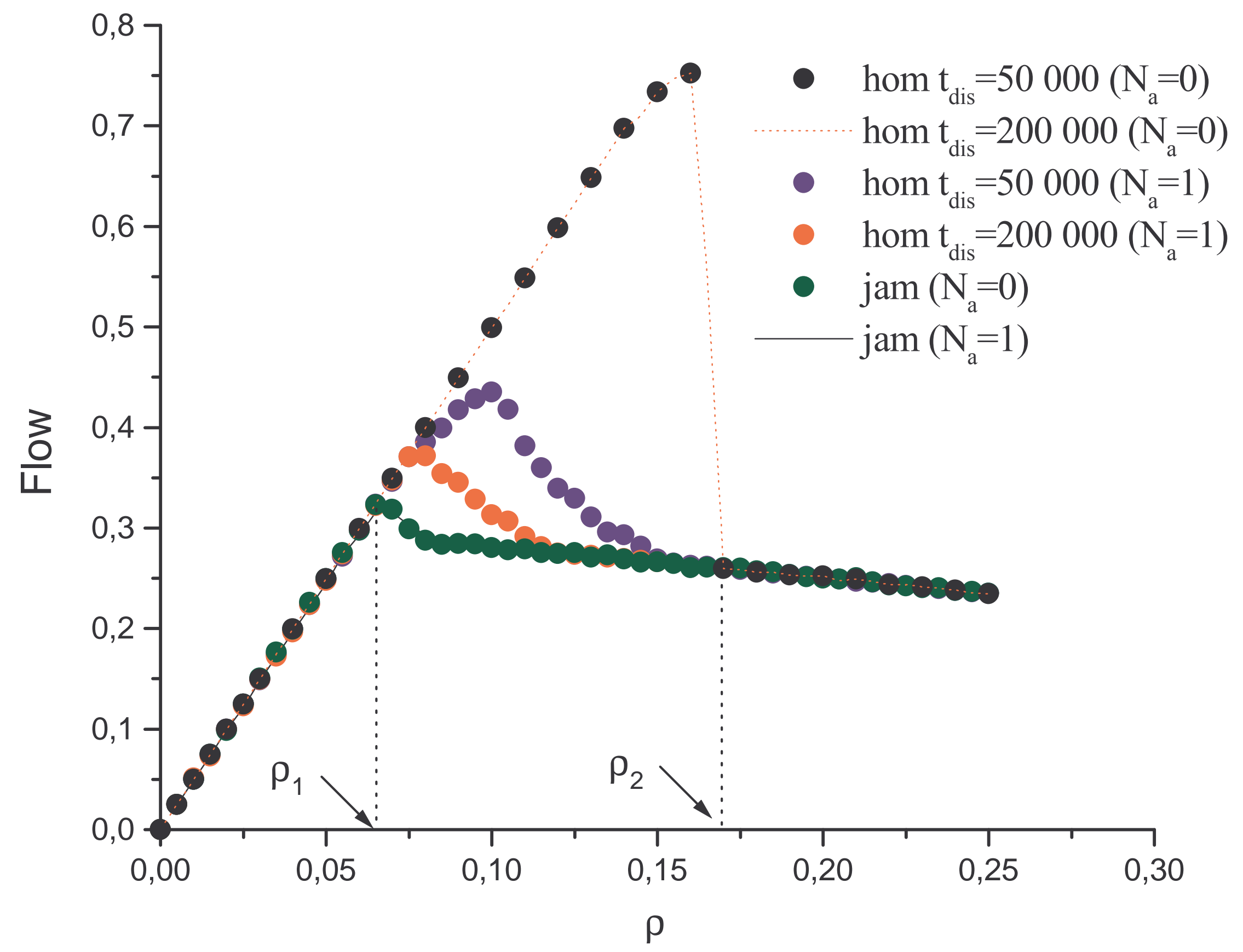




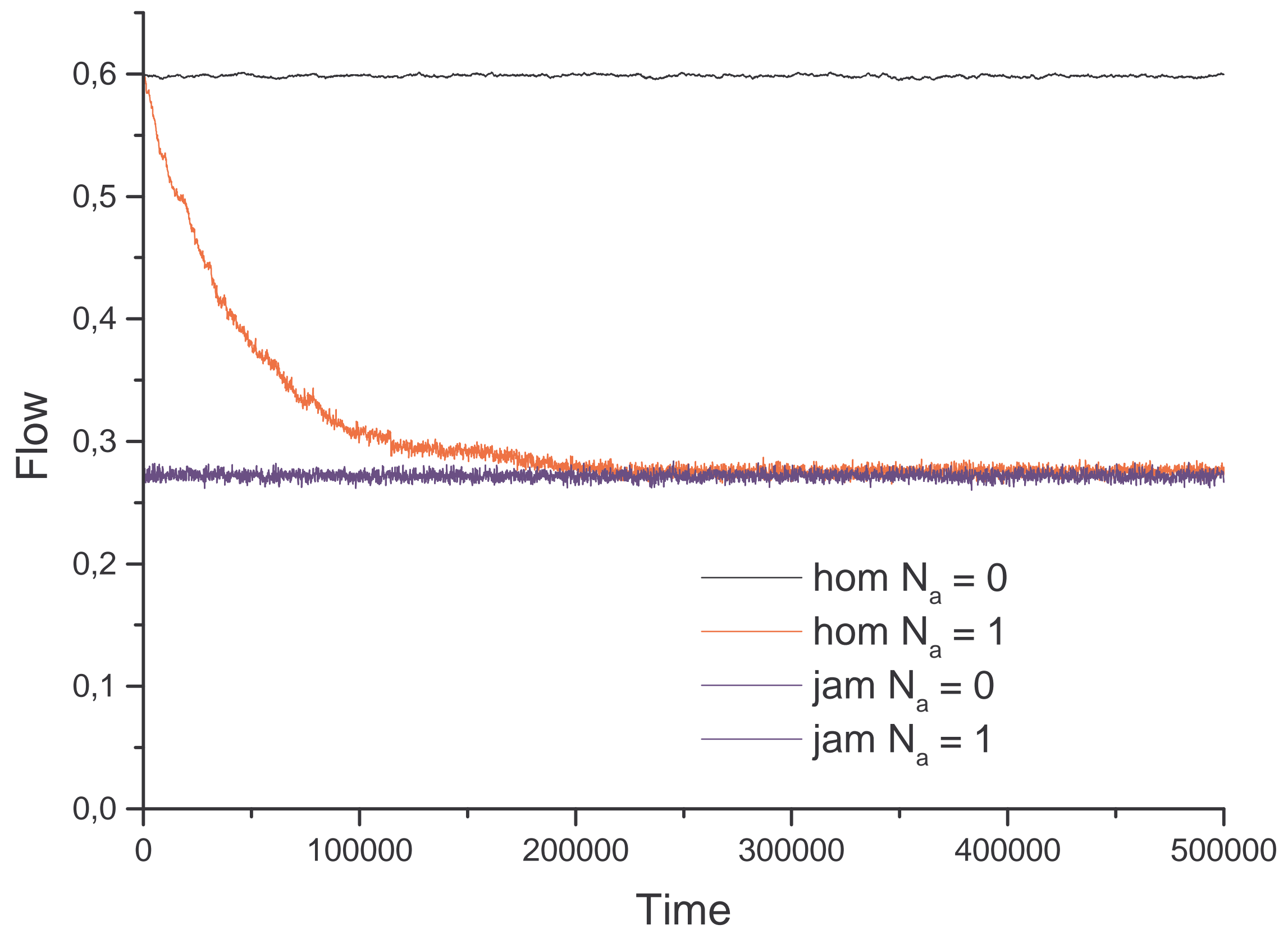




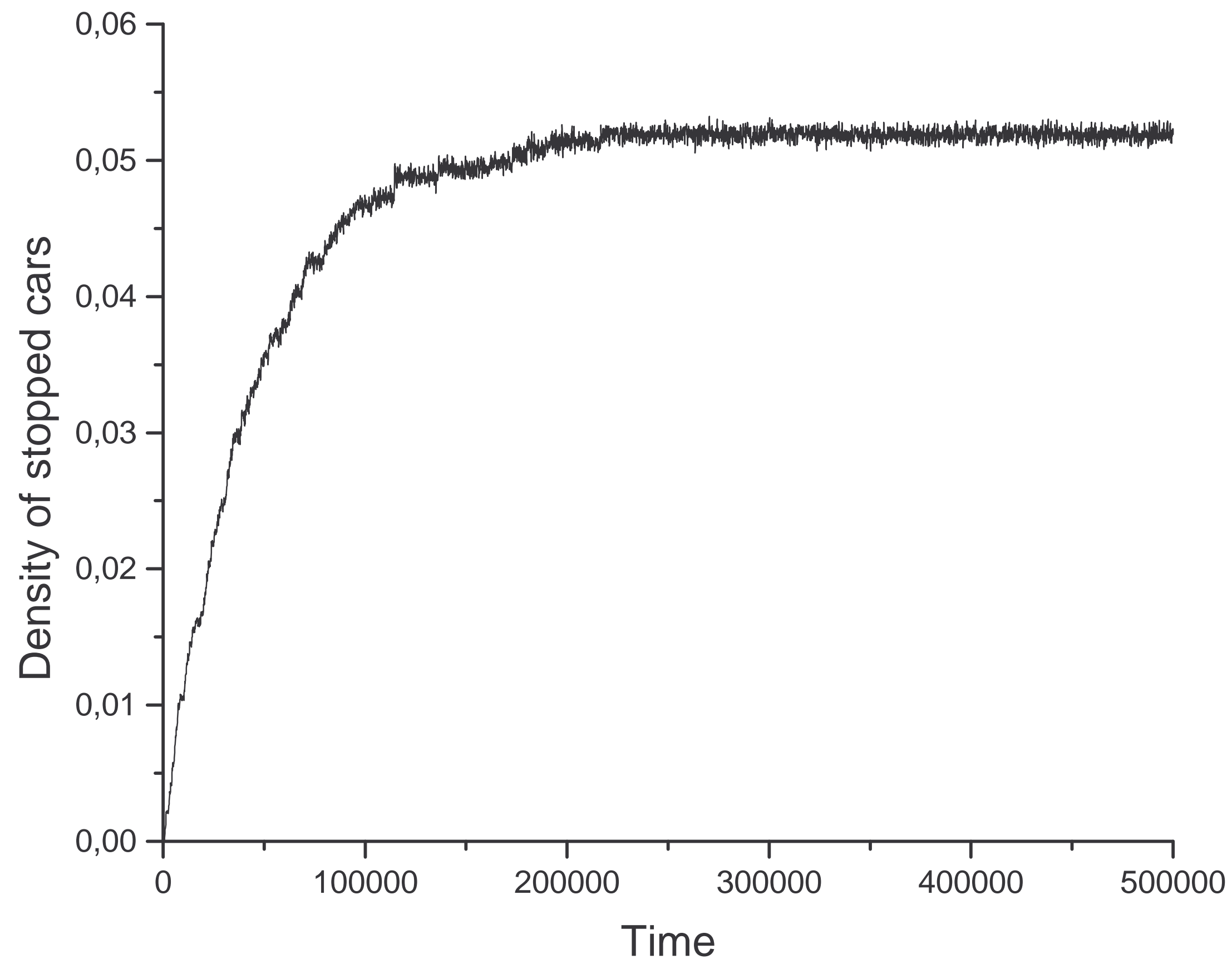




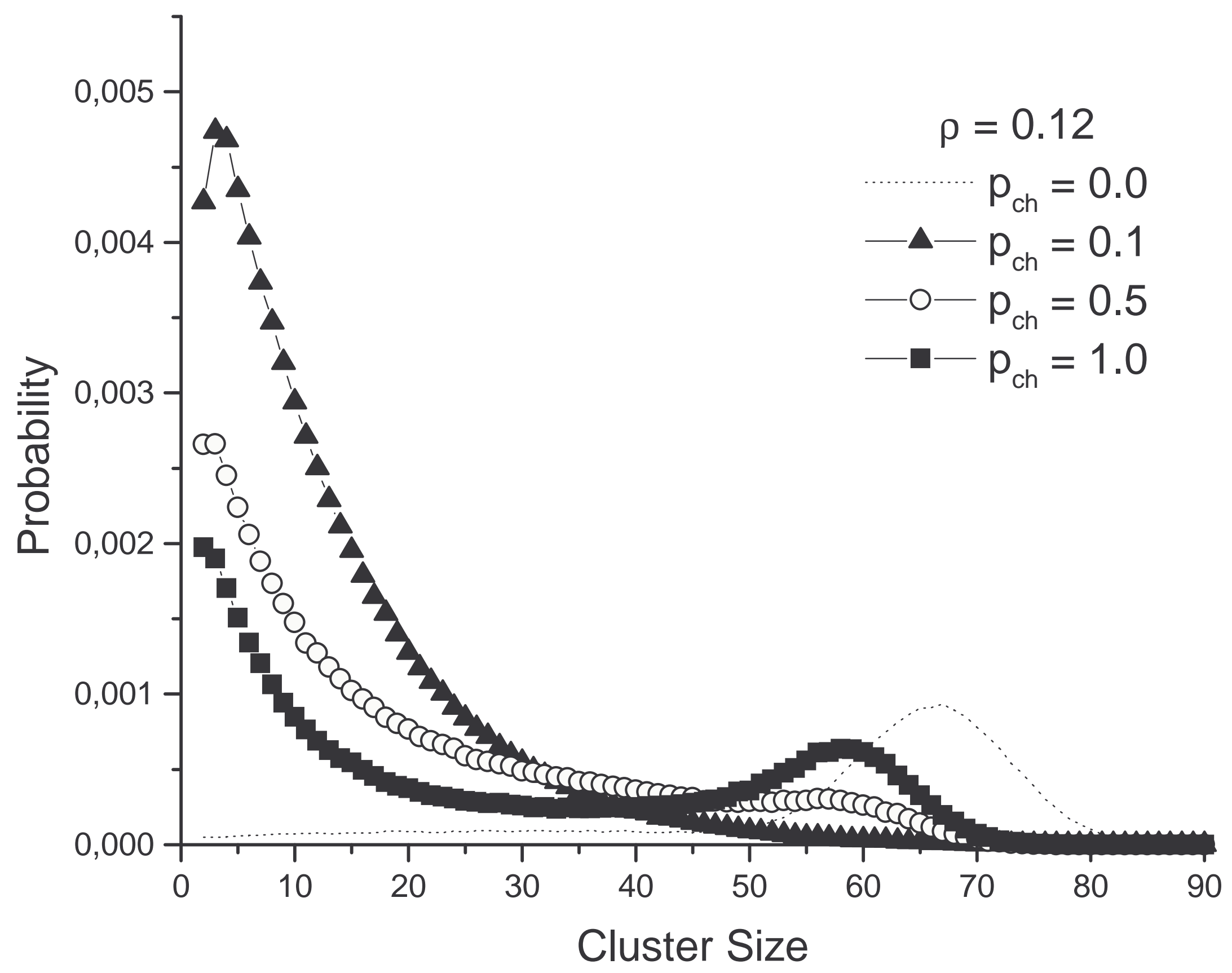




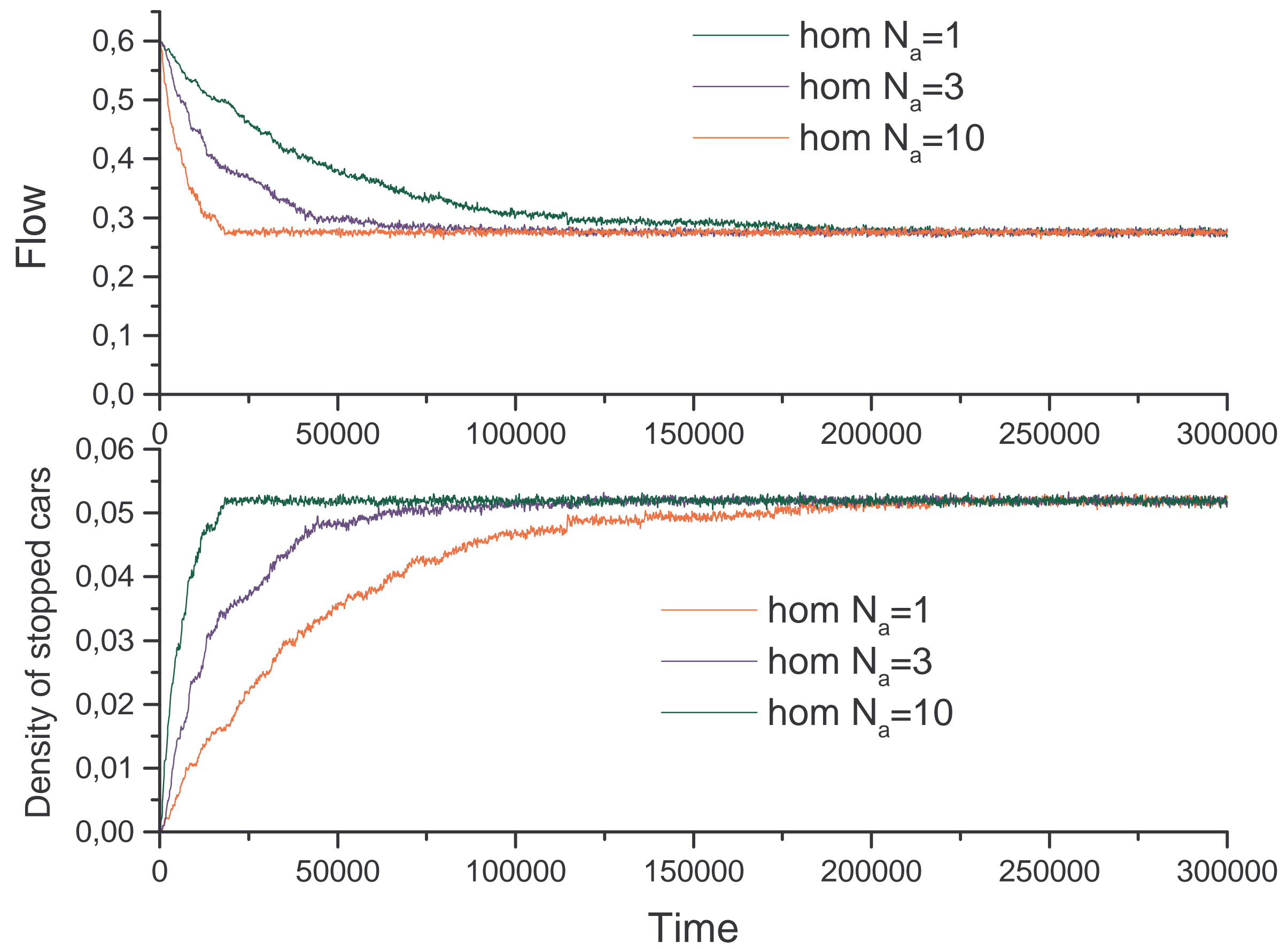




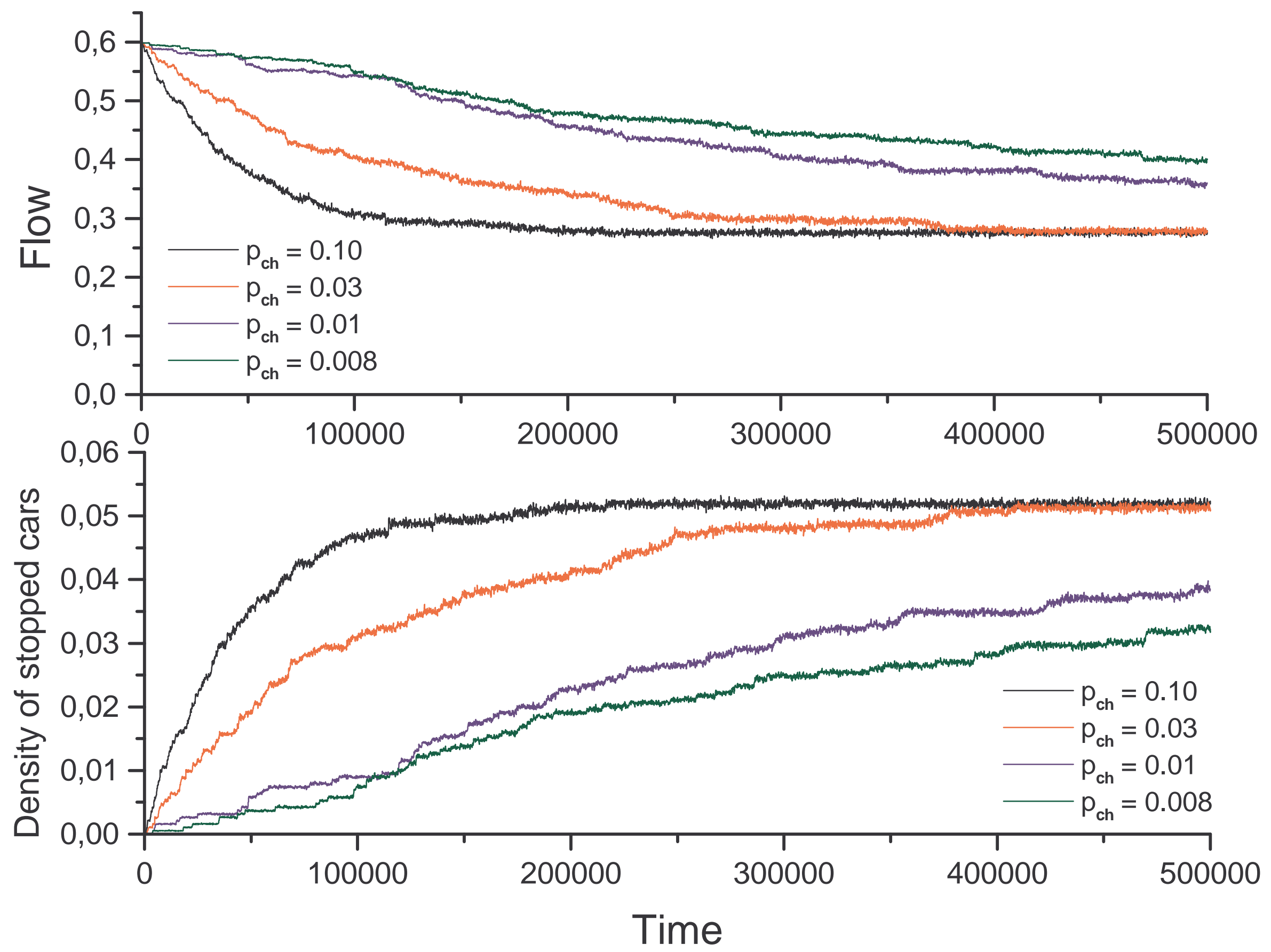




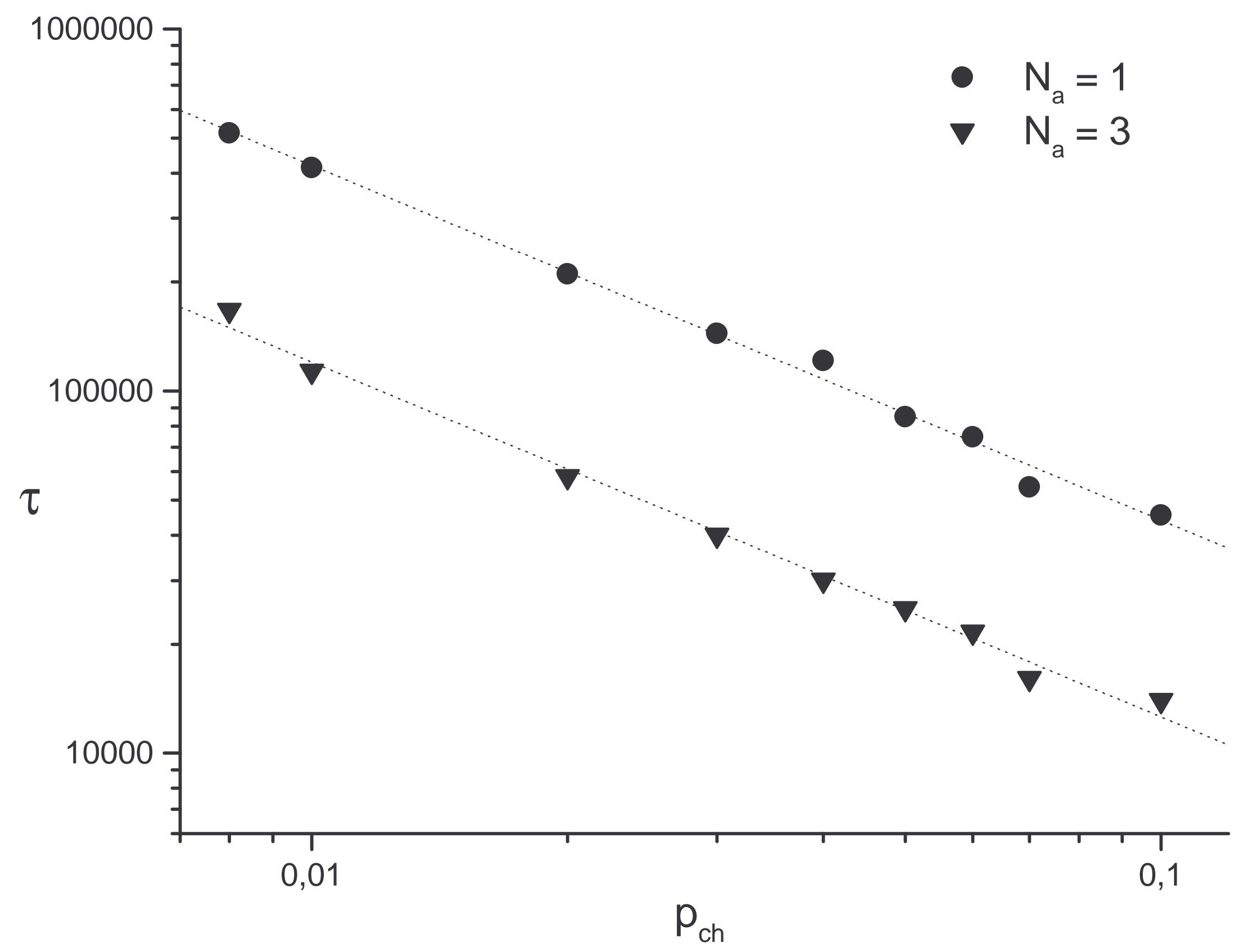

